\title{
Output Consensus Regulation for State-Unmeasurable Discrete-Time Multiagent Systems with External Disturbances
}

\author{
He Jiang and Dongsheng Yang \\ College of Information Science and Engineering, Northeastern University, Shenyang 110819, China \\ Correspondence should be addressed to Dongsheng Yang; yangdongsheng@mail.neu.edu.cn
}

Received 10 March 2015; Accepted 26 November 2015

Academic Editor: Zhan Shu

Copyright (c) $2015 \mathrm{H}$. Jiang and D. Yang. This is an open access article distributed under the Creative Commons Attribution License, which permits unrestricted use, distribution, and reproduction in any medium, provided the original work is properly cited.

\begin{abstract}
This paper deals with the output consensus regulation problem for discrete-time multiagent systems with state-unmeasurable agents and external disturbances under directed communication network topologies. Firstly, the mathematical model for the output consensus problem of discrete-time multiagent systems is deduced and formulated via making matrix transformation. Then, based on state observers, a novel output consensus protocol with dynamic compensator which is used as observer for the exosystem is proposed to solve this problem. Some knowledge of matrix theory and graph theory is introduced to design protocol parameters and the convergence of output consensus errors is proved. Finally, a numerical simulation example is shown to verify the effectiveness of the proposed protocol design.
\end{abstract}

\section{Introduction}

Recently, the researches on coordination problems of multiagent systems have received more and more attention from different fields, for the multiagent systems lie in almost everywhere in our daily life such as communication networks, power grids, social networks, and genetic regulatory networks. Therefore, multiagent systems have broad applications in many areas including formation control [1, 2], flocking [3, 4], unmanned air vehicles (UAVs) [5], distributed sensor networks [6], and attitude alignment [7]. In addition, the directed information flow among dynamic agents is based on the communication network topology digraph. Compared with the much similar interconnected systems [8-10], multiagent systems have advantages of reducing the cost and improving system efficiency as the distributed control protocols only require the information from the agent itself and its neighborhood agents through the communication digraph [11-14].

Among the coordination problems of multiagent systems, one of the most interesting topics is the leader-following problem, in which the exosystem named leader is a special agent whose motion is followed by the others named followers $[15,16]$. A leader-following consensus problem with coupling time delays was discussed by [17]. In [18], a frequency-domain leader-following consensus algorithm with communication input delays was proposed to find out the stability conditions. For the consensus problem under the case of an active leader and variable topologies, [19] gave an observer-based method for each agent to estimate the velocity of the leader. Reference [20] solves the leader-following formation problem of multirobot systems with switching interconnection topologies by using output feedback laws based on canonical internal model. An adaptive control law was designed to solve the robust leader-following formation problem in [21].

Output regulation for multiagent systems can be regarded as a special leader-following problem in which the output of the leader is tracked by the outputs of followers with rejection of the external disturbances generated by the exosystem [22, 23]. Therefore, the output regulation problem of multiagent systems is more challenging and receives much considerable attention in [24, 25]. In [26], the adaptive internal model technique was employed to achieve the disturbance rejection and output regulation. Reference [27] proposed a distributed coordinative control approach to solve the output regulation problem for a class of nonlinear multiagent systems. In [28], a novel dynamic distributed control protocol was designed by using output feedback laws to realize the cooperative output regulation of linear multiagent systems. The robust output 
regulation problem for a class of uncertain multiagent systems was addressed by using the internal model principle in [29]. Recently, the work of [30] studied the robust output regulation problem for a heterogeneous network of agents affected by parameter perturbations via constructing novel robust regulators. Based on a dynamic compensator, a cooperative output regulation approach for the continuous-time multiagent systems whose states are assumed measurable was given in [31].

There have been a great number of results for the output regulation problems of continuous-time multiagent systems, but few papers discuss discrete-time multiagent systems. It is known that the results of the continuous-time systems are hard to be applied to the practical applications, for the practical control systems are usually discrete-time sampling systems. In addition, the states of the multiagent systems in practical applications are usually unmeasurable, but the states are assumed measurable in many papers. Due to the communication topologies of multiagent systems, not all the agents can receive the information of the leader.

Therefore, according to the problems above, a novel output consensus protocol with a dynamic compensator based on state observers will be presented to solve the output consensus regulation problem for discrete-time multiagent systems with state-unmeasurable agents and external disturbances under directed communication network topologies.

The rest of the paper is organized as follows. In Section 2, some basic knowledge of graph theory for multiagent systems is introduced, and the mathematical model for the output consensus problem of discrete-time multiagent systems is formulated. Section 3 presents the main results of output consensus protocol design and output consensus error convergence proof. In Section 4, a numerical simulation example will be given to verify the effectiveness of the proposed control protocol. Finally, conclusions are drawn in Section 5.

The following notations are used throughout this paper. $R$ and $C$ denote the sets of the real numbers and complex numbers, respectively. $R^{m \times n}$ denotes the set of matrices with $m$ rows and $n$ columns. For a given matrix $A, A^{T}$ is the transpose of $A$. For a given matrix $B \in R^{n \times n}, B>0$ means that $B$ is a positive definite matrix. $I_{N}$ denotes the $N$-dimension unit matrix, and $1_{N}$ represents $\left(\begin{array}{llll}1 & 1 & \cdots & 1\end{array}\right)^{T}$ with dimension $N$. $\otimes$ denotes the Kronecker product and the Kronecker sum of $A^{m \times m}$ and $B^{n \times n}$ can be defined as $A \oplus B=\left(A \otimes I_{n}\right)+\left(B \otimes I_{m}\right)$.

\section{Preliminaries and Problem Formulation}

This section introduces some basic knowledge and concepts of graph theory which will be used throughout this paper and formulates the mathematical model for the output consensus problem of discrete-time multiagent systems.

2.1. Algebraic Graph Theory. The topology of a communication network for a multiagent system can be expressed by a directed graph. A weighted digraph $\mathscr{G}=(\mathscr{V}, \mathscr{E}, \mathscr{A})$ consists of a vertex set $\mathscr{V}=\left\{v_{1}, v_{2}, \ldots, v_{n}\right\}$, an edge set $\mathscr{E}=\left\{e_{i j}=\right.$ $\left.\left(v_{i}, v_{j}\right)\right\} \in \mathscr{V} \times \mathscr{V}$, and a weighted adjacency matrix $\mathscr{A}=\left[a_{i j}\right]$ with nonnegative adjacent elements $a_{i j}$. If $\left(v_{i}, v_{j}\right) \in \mathscr{E}$, then $a_{i j}>0$; if $\left(v_{i}, v_{j}\right) \notin \mathscr{E}$, then $a_{i j}=0$. For all $i=1,2, \ldots, n$, $a_{i i}=0$. If and only if $i$ th agent can receive information from $j$ th agent directly, then $\left(v_{i}, v_{j}\right) \in \mathscr{E}$. The set of neighbours of the node $v_{i}$ can be defined as $\mathcal{N}_{i}=\left\{v_{j} \in \mathscr{V}:\left(v_{i}, v_{j}\right) \in \mathscr{E}\right\}$. The in-degree and out-degree of the node $v_{i}$ can be given by

$$
\begin{gathered}
\operatorname{deg}_{\text {in }}\left(v_{i}\right)=\sum_{j=1}^{n} a_{j i}, \\
\operatorname{deg}_{\text {out }}\left(v_{i}\right)=\sum_{j=1}^{n} a_{i j} .
\end{gathered}
$$

The degree matrix $\mathscr{D}=\operatorname{diag}\left\{d_{1}, d_{2}, \ldots, d_{n}\right\}$ is a diagonal matrix, whose elements are composed of $d_{i}=\operatorname{deg}_{\text {out }}\left(v_{i}\right)$. The Laplacian matrix $\mathscr{L}=\mathscr{D}-\mathscr{A}=\left[l_{i j}\right]_{n \times n}$ consists of $l_{i j}=-a_{i j}$ and $l_{i i}=\sum_{j=1}^{n} a_{i j}$. The exosystem which is usually defined as node 0 only sends information to other agents without receiving any information from others. This means that $a_{01}=a_{02}=\cdots=a_{0 n}=0$, and $a_{i 0}>0$ if the $i$ th agent can receive information from the exosystem.

Remark 1. Laplacian matrix has all row sums equal to zero, which will be used for the following derivation later.

2.2. Problem Formulation. Consider a multiagent system consisting of $N$ nonidentical discrete-time agents with external disturbances. The dynamics of agents can be described by

$$
\begin{aligned}
& x_{i}^{\dagger}=A_{i} x_{i}+B_{i} u_{i}+E_{i} w \\
& y_{i}=C_{i} x_{i}, \quad i=1,2, \ldots, N,
\end{aligned}
$$

where $x_{i} \in R^{n}$ is the state of each agent and $x_{i}^{\dagger}$ denotes $x_{i}(k+1) ; u_{i} \in R^{m_{i}}$ is the output consensus regulation protocol; $E_{i} w \in R^{n}$ denotes the external disturbance of each agent; and $y_{i} \in R^{p}$ is the output of each agent.

A reference system, which can be also called leader or exosystem, is defined as

$$
\begin{aligned}
& w^{\dagger}=S w, \quad w(0)=w_{0} \\
& y_{r}=F w,
\end{aligned}
$$

where $w \in R^{q}$ is the state of the leader and $w^{\dagger}$ denotes $w(k+$ 1); $y_{r} \in R^{p}$ is the reference output signal for the multiagent system.

The local output consensus error $e_{i} \in R^{n}$ can be defined as

$$
e_{i}=y_{i}-y_{r}, \quad i=1,2, \ldots, N
$$
by

The state observer for the multiagent system can be given

$$
\widehat{x}_{i}^{\dagger}=A_{i} \widehat{x}_{i}+B_{i} u_{i}+E_{i} w+L_{i}\left(C_{i} \widehat{x}_{i}-C_{i} x_{i}\right),
$$

where $\widehat{x}_{i}^{\dagger}$ denotes $\widehat{x}_{i}(k+1)$ and $L_{i}$ is the gain matrix for the state observer which will be designed later. 
In the multiagent system, not all the agents can directly receive information from the leader. For this situation, a dynamic compensator, which can be regarded as a distributed observer for the leader, is introduced as

$$
\xi_{i}^{\dagger}=S \xi_{i}+\phi\left(\sum_{j \in N_{i}} a_{i j}\left(\xi_{i}-\xi_{j}\right)+a_{i 0}\left(\xi_{i}-w\right)\right),
$$

where $\xi_{i} \in R^{q}$, $\xi_{i}^{\dagger}$ denotes $\xi_{i}(k+1)$, and $\phi$ is a constant parameter.

Based on the state observer and dynamic compensator, the output consensus protocol can be given by

$$
u_{i}=K_{1 i} \widehat{x}_{i}+K_{2 i} \xi_{i}, \quad i=1,2, \ldots, N,
$$

where $K_{1 i} \in R^{m \times n}$ and $K_{2 i} \in R^{m \times q}$ are the gain matrices which will be designed later.

Let $\bar{x}=\left[x_{1}^{T}, x_{2}^{T}, \ldots, x_{N}^{T}, \widehat{x}_{1}^{T}, \widehat{x}_{2}^{T}, \ldots, \widehat{x}_{N}^{T}\right]^{T}, \bar{\xi}=\left[\xi_{1}^{T}, \xi_{2}^{T}, \ldots\right.$, $\left.\xi_{N}^{T}\right]^{T}$, and $\bar{w}=1_{N} \otimes w$. Then one can obtain global model as follows:

$$
\begin{aligned}
& \bar{x}^{\dagger}=\bar{A} \bar{x}+\bar{B} \bar{\xi}+\bar{E} \bar{w} \\
& \bar{\xi}^{\dagger}=(\phi H \oplus S) \bar{\xi}-\phi\left(A_{0} \otimes I_{q}\right) \bar{w},
\end{aligned}
$$

where $A_{0}=\operatorname{diag}\left\{a_{10}, a_{20}, \ldots, a_{N 0}\right\}$ and $H=\mathscr{L}+A_{0} . \mathscr{L}$ is the Laplacian matrix of the digraph, and

$$
\begin{aligned}
& \bar{A}=\left(\begin{array}{cc}
\bar{A}_{*} & \bar{B}_{*} \bar{K}_{1} \\
-\bar{L} \bar{C} & \bar{A}_{*}+\bar{B}_{*} \bar{K}_{1}+\bar{L} \bar{C}
\end{array}\right), \\
& \bar{B}=\left(\begin{array}{l}
\bar{B}_{*} \bar{K}_{2} \\
\bar{B}_{*} \bar{K}_{2}
\end{array}\right), \\
& \bar{E}=\left(\begin{array}{l}
\bar{E}_{*} \\
\bar{E}_{*}
\end{array}\right)
\end{aligned}
$$

with

$$
\begin{aligned}
\bar{A}_{*} & =\operatorname{diag}\left\{A_{1}, A_{2}, \ldots, A_{N}\right\}, \\
\bar{B}_{*} & =\operatorname{diag}\left\{B_{1}, B_{2}, \ldots, B_{N}\right\}, \\
\bar{C} & =\operatorname{diag}\left\{C_{1}, C_{2}, \ldots, C_{N}\right\}, \\
\bar{E}_{*} & =\operatorname{diag}\left\{E_{1}, E_{2}, \ldots, E_{N}\right\}, \\
\bar{L} & =\operatorname{diag}\left\{L_{1}, L_{2}, \ldots, L_{N}\right\}, \\
\bar{K}_{1} & =\operatorname{diag}\left\{K_{11}, K_{12}, \ldots, K_{1 N}\right\}, \\
\bar{K}_{2} & =\operatorname{diag}\left\{K_{21}, K_{22}, \ldots, K_{2 N}\right\} .
\end{aligned}
$$

Let $\widetilde{x}=\left[\bar{x}^{T}, \bar{\xi}^{T}\right]^{T}, \widetilde{A}=\left(\begin{array}{cc}\bar{A} & \bar{B} \\ 0 & \phi H \oplus S\end{array}\right)$, and $\widetilde{E}=\left(\begin{array}{c}\bar{E} \\ -\phi\left(A_{0} \otimes I_{q}\right)\end{array}\right)$.

Then the global model can be rewritten as

$$
\widetilde{x}^{\dagger}=\widetilde{A} \widetilde{x}+\widetilde{E} \bar{w},
$$

where $\tilde{x}^{\dagger}$ denotes $\widetilde{x}(k+1)$.
Problem 2. The output consensus regulation problem can be defined as follows:

(1) The global model is asymptotically stable without external disturbance; that is, $\widetilde{A}=\left(\begin{array}{ccc}\bar{A} & \bar{B} \\ 0 & \phi H \oplus S\end{array}\right)$ is Schur stable.

(2) For all the initial conditions $x_{i}(0)$ and $w(0)$, the output consensus errors satisfy

$$
\begin{aligned}
\lim _{k \rightarrow \infty} e_{i}(k)=\lim _{k \rightarrow \infty}\left(C_{i} x_{i}(k)-F w(k)\right) & =0, \\
i & =1,2, \ldots, N .
\end{aligned}
$$

\section{Main Results}

In this section, two lemmas are introduced and two theorems are proposed to design the control parameters $\left(K_{1 i}, K_{2 i}, \phi, L_{i}\right)$ of the output consensus protocol and to prove the convergence of the output consensus errors, respectively.

Firstly, the gain matrices $K_{1 i}$ and $L_{i}$ are designed by the following lemma.

Lemma 3 (see [32]). Given the stabilizable pair $(A, B)$, one gets the following discrete-time algebraic Riccati equation:

$$
A^{T} P A-P-A^{T} P B\left(B^{T} P B\right)^{-1} B^{T} P A+Q=0
$$

with a unique solution $P=P^{T}>0, Q=Q^{T}>0$, and $Q=\widetilde{Q} \widetilde{Q}$ for symmetric matrix $\widetilde{Q}>0$. Matrix $A+s B K(K=$ $\left.-\left(B^{T} P B\right)^{-1} B^{T} P A, s \in C\right)$ is Schur stable with all its eigenvalues in the open unit disc if and only if s lies in the stability region:

$$
\Psi=\left\{s \in C:|s-1|^{2}<\theta\right\},
$$

where

$$
\theta=\frac{1}{\lambda_{\max }\left[\widetilde{Q}^{-1} A^{T} P B\left(B^{T} P B\right)^{-1} B^{T} P A \widetilde{Q}^{-1}\right]} .
$$

Assumption 4. The pairs $\left(A_{i}, B_{i}\right)$ are controllable and the pairs $\left(C_{i}, A_{i}\right)$ are detectable, $i=1,2, \ldots, N$.

If Assumption 4 holds, the gain matrices $K_{1 i}$ and $L_{i}$ can be easily designed to make $A_{i}+B_{i} K_{1 i}$ and $A_{i}+L_{i} C_{i}$ Schur stable according to Lemma 3 .

Remark 5. It is known that the detectable pairs $\left(C_{i}, A_{i}\right)$ are equivalent to the controllable pairs $\left(A_{i}^{T}, C_{i}^{T}\right)$. Then Lemma 3 can be directly used to design $L_{i}$ to make $A_{i}+L_{i} C_{i}$ Schur stable, which have all its eigenvalues in the open unit disc.

Let $\widetilde{e}_{i}^{\dagger}=x_{i}^{\dagger}-\widehat{x}_{i}^{\dagger}$; then one can obtain by direct calculation the following:

$$
\begin{aligned}
\tilde{e}_{i}^{\dagger}= & x_{i}^{\dagger}-\widehat{x}_{i}^{\dagger} \\
= & A_{i} x_{i}+B_{i} u_{i}+E_{i} w \\
& -\left(A_{i} \widehat{x}_{i}+B_{i} u_{i}+E_{i} w+L_{i}\left(C_{i} \widehat{x}_{i}-C_{i} x_{i}\right)\right) \\
= & \left(A_{i}+L_{i} C_{i}\right) \tilde{e}_{i} .
\end{aligned}
$$


Since $A_{i}+L_{i} C_{i}$ are Schur stable, $\lim _{k \rightarrow \infty} \widetilde{e}_{i}(k)=0$; that is, $\widehat{x}(k) \rightarrow x(k)$, as $k \rightarrow \infty$.

Theorem 6. If Assumption 4 holds, then matrix $\bar{A}$ is Schur stable.

Proof. Matrix $\bar{A}$ is similar to matrix $\bar{A}_{s}=T^{-1} \bar{A} T$. The transformed matrix can be defined as $T=\left(\begin{array}{ll}I & 0 \\ I & I\end{array}\right)$. Therefore, $\bar{A}_{s}=T^{-1} \bar{A} T=\left(\begin{array}{cc}\bar{A}_{*}+\bar{B}_{*} \bar{K}_{1} & \bar{B}_{*} \bar{K}_{1} \\ 0 & \bar{A}_{*}+\bar{L} \bar{C}\end{array}\right)$.

It is obvious that $\bar{A}_{*}+\bar{B}_{*} \bar{K}_{1}$ and $\bar{A}_{*}+\bar{L} \bar{C}$ are Schur stable because $A_{i}+B_{i} K_{1 i}$ and $A_{i}+L_{i} C_{i}$ are designed to be Schur stable. Thus, $\bar{A}_{s}$ is Schur stable; that is, $\bar{A}$ is Schur stable.

The proof is completed.

Next, the constant parameter $\phi$ will be designed by the following lemma.

Lemma 7 (see [33]). Given the matrices $A \in R^{n \times n}$ and $B \in$ $R^{m \times m}$, the eigenvalues of $A \oplus B$ are $\lambda_{i}+\mu_{j}, i=1,2, \ldots, n, j=$ $1,2, \ldots, m$, where $\lambda_{i}$ and $\mu_{j}$ are the eigenvalues of $A$ and $B$, respectively.

According to Lemma 7, the eigenvalues of matrix $\phi H \oplus S$ can be given by

$$
\begin{aligned}
\lambda & (\phi H \oplus S) \\
& =\left\{\phi \lambda_{i}(H)+\lambda_{j}(S) \mid i=1 \cdots N, j=1 \cdots q\right\} .
\end{aligned}
$$

One can find out a proper constant parameter $\phi$ to make the magnitude of $\phi \lambda_{i}(H)+\lambda_{j}(S)$ less than 1 , that is, to make matrix $\phi H \oplus S$ Schur stable.

Theorem 8. Under Assumption 4, if there exist matrices $\eta_{i}$ and $\zeta_{i}, i=1,2, \ldots, N$, satisfying the following equations:

$$
\begin{aligned}
\eta_{i} S & =A_{i} \eta_{i}+B_{i} \zeta_{i}+E_{i} \\
0 & =C_{i} \eta_{i}-F,
\end{aligned}
$$

then the output consensus protocol (7) solves the output consensus problem for the discrete-time multiagent system with external disturbances and heterogenous agents whose states are unmeasurable.

Proof. Let $z_{i}=x_{i}-\eta_{i} w, \widehat{z}_{i}=\widehat{x}_{i}-\eta_{i} w, \bar{z}=\left[z_{1}^{T}, z_{2}^{T}, \ldots, z_{N}^{T}\right.$, $\left.\widehat{z}_{1}^{T}, \widehat{z}_{2}^{T}, \ldots, \widehat{z}_{N}^{T}\right]^{T}$, and $\bar{\eta}=\operatorname{diag}\left\{\eta_{1}, \eta_{2}, \ldots, \eta_{N}\right\} ; \bar{z}$ can be expressed by

$$
\bar{z}=\bar{x}-1_{2} \otimes(\bar{\eta} \bar{w})
$$

According to (19), one can obtain

$$
\bar{z}^{\dagger}=\bar{x}^{\dagger}-1_{2} \otimes\left(\bar{\eta} \bar{w}^{\dagger}\right)
$$

Since $\bar{w}^{\dagger}=\left(I_{N} \otimes S\right) \bar{w},(20)$ can be rewritten as

$$
\bar{z}^{\dagger}=\bar{A} \bar{z}+\bar{B} \bar{\xi}+\varepsilon \bar{w}
$$

where $\varepsilon=\bar{A}\left(1_{2} \otimes \bar{\eta}\right)+\bar{E}-\left(1_{2} \otimes \bar{\eta}\right)\left(I_{N} \otimes S\right)$. Next, the gain matrices $K_{2 i}$ are designed as

$$
K_{2 i}=\zeta_{i}-K_{1 i} \eta_{i}
$$

Then (18) can be expressed as

$$
\eta_{i} S=\left(A_{i}+B K_{1 i}\right) \eta_{i}+B_{i} K_{2 i}+E_{i}
$$

Since

$$
\begin{aligned}
1_{2} & \otimes\left(\bar{A}_{*}+\bar{B}_{*} \bar{K}_{1}\right) \bar{\eta}=\left(\begin{array}{l}
\left(\bar{A}_{*}+\bar{B}_{*} \bar{K}_{1}\right) \bar{\eta} \\
\left(\bar{A}_{*}+\bar{B}_{*} \bar{K}_{1}\right) \bar{\eta}
\end{array}\right) \\
& =\left(\begin{array}{cc}
\bar{A}_{*} & \bar{B}_{*} \bar{K}_{1} \\
-\bar{L} \bar{C} & \bar{A}_{*}+\bar{B}_{*} \bar{K}_{1}+\bar{L} \bar{C}
\end{array}\right)\left(\begin{array}{l}
\bar{\eta} \\
\bar{\eta}
\end{array}\right)=\bar{A}\left(1_{2} \otimes \bar{\eta}\right),
\end{aligned}
$$

then one can attain

$$
\begin{aligned}
\left(1_{2}\right. & \otimes \bar{\eta})\left(I_{N} \otimes S\right)=1_{2} \otimes\left(\bar{\eta}\left(I_{N} \otimes S\right)\right) \\
& =1_{2} \otimes\left(\left(\bar{A}_{*}+\bar{B}_{*} \bar{K}_{1}\right) \bar{\eta}+\bar{B}_{*} \bar{K}_{2}+\bar{E}_{*}\right) \\
& =\bar{A}\left(1_{2} \otimes \bar{\eta}\right)+\bar{B}+\bar{E}
\end{aligned}
$$

Substituting (25) into (21), then (21) can be rewritten as

$$
\bar{z}^{\dagger}=\bar{A} \bar{z}+\bar{B}(\bar{\xi}-\bar{w})
$$

Since $\mathscr{L}$ is the Laplacian matrix of the digraph for the multiagent systems, then $\phi\left(\mathscr{L} \otimes I_{q}\right) \bar{w}=0$ which have been mentioned in Remark 1. Therefore, one gets

$$
\begin{aligned}
\bar{\xi}^{\dagger}-\bar{w}^{\dagger}= & (\phi H \oplus S) \bar{\xi}-\phi\left(A_{0} \otimes I_{q}\right) \bar{w}-\left(I_{N} \otimes S\right) \bar{w} \\
= & (\phi H \oplus S) \bar{\xi} \\
& -\left(\phi\left(A_{0}+\mathscr{L}\right) \otimes I_{q}+\left(I_{N} \otimes S\right)\right) \bar{w} \\
= & (\phi H \oplus S) \bar{\xi}-\left(\left(\phi H \otimes I_{q}\right)+\left(I_{N} \otimes S\right)\right) \bar{w} \\
= & (\phi H \oplus S)(\bar{\xi}-\bar{w}) .
\end{aligned}
$$

Let $\mathscr{Z}=\left(\bar{z}^{T},(\bar{\xi}-\bar{w})^{T}\right)^{T}$; combining (26) and (27), one has

$$
\mathscr{Z}^{\dagger}=\widetilde{A} \mathscr{Z} \text {. }
$$

By using the proposed Theorem 6 and Lemma 7, $\bar{A}$ and $\phi H \oplus$ $S$ are designed to be Schur stable; that is, $\widetilde{A}$ which has been defined before is Schur stable. Therefore, $\mathscr{Z} \rightarrow 0$ as $k \rightarrow 0$; that is, $\lim _{k \rightarrow \infty} z_{i}(k)=0, i=1,2, \ldots, N$.

Since $z_{i}=x_{i}-\eta_{i} w$, the output errors can be expressed as

$$
e_{i}=C_{i}\left(z_{i}+\eta_{i} w\right)-F w=C_{i} z_{i}+\left(C_{i} \eta_{i}-F\right) w=C_{i} z_{i}
$$

As $\lim _{k \rightarrow \infty} z_{i}(k)=0$, thus, $\lim _{k \rightarrow \infty} e_{i}(k)=0$. The proof is completed. 
Remark 9. Compared with the traditional methods of using LMI (Linear Matrix Inequality) and Lyapunov techniques, the proposed approach in this paper only needs to solve some simple linear matrix equations (18) and discrete-time algebraic Riccati equations in Lemma 3 to obtain the control parameters, which successfully reduces the expensive computation and computational complexity.

Remark 10. It is known that there are a great deal of works [24-29] investigating the output regulation problems for continuous-time multiagent systems. However, these approaches under continuous-time cases are usually invalid for the realworld discrete-time sampling systems. Therefore, the proposed scheme which studies the discrete-time cases in this paper makes great sense for the real-world applications. Furthermore, this paper successfully solves the output regulation problems with unmeasurable states in contrast to the limit of measurable states in the previous works [31,32], which will be very useful for the practical engineering applications.

\section{Numerical Simulation Example}

In this section, a numerical simulation example will be taken to verify the effectiveness of the proposed output consensus protocol for the discrete-time multiagent systems. For the sake of simplicity and without loss of generality, consider a multiagent system consisting of four agents with

$$
\begin{aligned}
A_{i} & =\left(\begin{array}{cc}
0.5 & 0 \\
1 & 0.3
\end{array}\right), \\
B_{i} & =\left(\begin{array}{l}
1 \\
0
\end{array}\right), \\
C_{i} & =\left(\begin{array}{ll}
0 & 1
\end{array}\right), \\
E_{i} & =\left(\begin{array}{ll}
i & -i \\
0 & 0
\end{array}\right),
\end{aligned}
$$

$$
i=1,2,3,4
$$

and the leader with

$$
\begin{aligned}
& S=\left(\begin{array}{cc}
1.5 & 0 \\
1 & 0.3
\end{array}\right), \\
& F=\left(\begin{array}{ll}
0 & 1
\end{array}\right) .
\end{aligned}
$$

According to the digraph for the communication topology of the multiagent system shown in Figure 1, only node

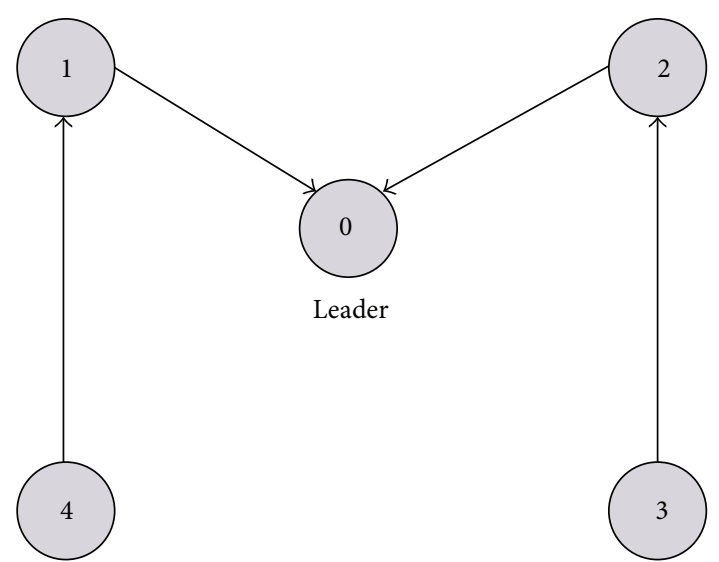

FIGURE 1: The digraph for the communication topology of the multiagent system.

1 and node 2 can receive information from the leader. The Laplacian matrix $\mathscr{L}$ and matrix $H$ can be given by

$$
\begin{aligned}
& \mathscr{L}=\left(\begin{array}{cccc}
0 & 0 & 0 & 0 \\
0 & 0 & 0 & 0 \\
0 & -1 & 1 & 0 \\
-1 & 0 & 0 & 1
\end{array}\right), \\
& H=\left(\begin{array}{cccc}
1 & 0 & 0 & 0 \\
0 & 1 & 0 & 0 \\
0 & -1 & 1 & 0 \\
-1 & 0 & 0 & 1
\end{array}\right)
\end{aligned}
$$

By using Lemma 3, $K_{1 i}=\left(\begin{array}{ll}-0.65 & -0.05\end{array}\right)$ and $L_{i}=$ $(-0.13-0.56)^{T}$ can be designed such that $A_{i}+B_{i} K_{1 i}$ and $A_{i}+L_{i} C_{i}$ are, respectively, Schur stable.

According to Lemma 7, there exists constant parameter $\phi=-1$ such that $\phi H \oplus S$ is Schur stable, which has all its eigenvalues in the open unit disc.

By solving (18), one can obtain $\eta_{i}=\left(\begin{array}{ll}1 & 0 \\ 0 & 1\end{array}\right)$, and $\zeta_{i}=$ $(1-i i), i=1,2,3,4$. Then $K_{2 i}=\zeta_{i}-K_{1 i} \eta_{i}=$ $(1.65-i \quad i+0.05)$.

The simulation results are shown in Figure 2. The output consensus errors converge to zero asymptotically, and finally the whole multiagent system achieves output consensus.

\section{Conclusion}

The output consensus regulation problem for discrete-time multiagent systems with state-unmeasurable agents and external disturbances under directed communication network topologies has been studied in this paper. A directed graph is used to describe the information exchange among the leader and the followers. Based on state observers, a novel output consensus protocol with dynamic compensator has been proposed to solve this problem. Compared with other control protocol designs, we take the case of unmeasurable agents and external disturbances into consideration and apply our 


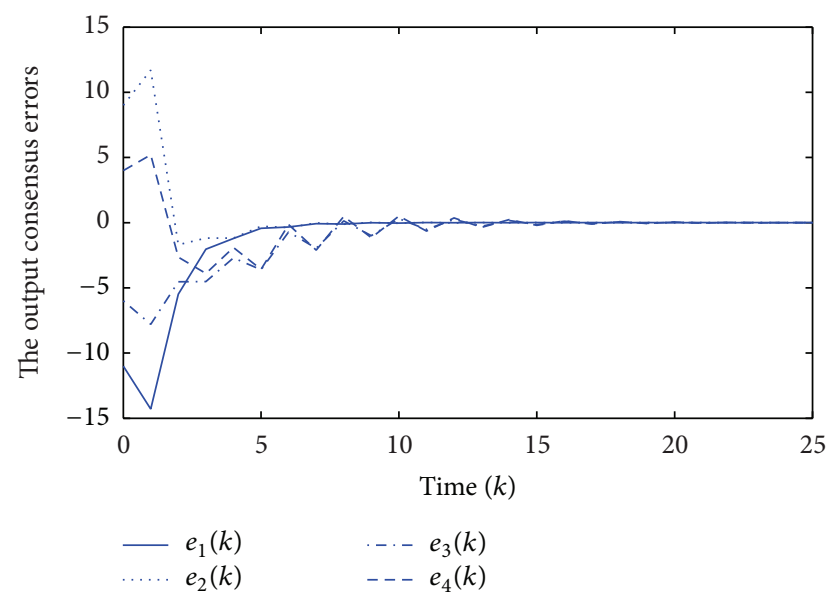

FIgURE 2: The output consensus errors.

results to the discrete-time multiagent systems. Therefore, our proposed results will be very useful for practical engineering applications.

\section{Conflict of Interests}

The authors declare that there is no conflict of interests regarding the publication of this paper.

\section{Acknowledgments}

This work was supported by the National Natural Science Foundation of China (61273029), the Program for New Century Excellent Talents in University, China (NCET-12-0106), and the Basic Scientific Research Funding of Northeastern University (N130504004).

\section{References}

[1] D. Barbucha, "Search modes for the cooperative multi-agent system solving the vehicle routing problem," Neurocomputing, vol. 88, pp. 13-23, 2012.

[2] J. A. Fax and R. M. Murray, "Information flow and cooperative control of vehicle formations," IEEE Transactions on Automatic Control, vol. 49, no. 9, pp. 1465-1476, 2004.

[3] Y. Hong, L. Gao, D. Cheng, and J. Hu, "Lyapunov-based approach to multiagent systems with switching jointly connected interconnection," IEEE Transactions on Automatic Control, vol. 52, no. 5, pp. 943-948, 2007.

[4] A. Jadbabaie, J. Lin, and A. S. Morse, "Coordination of groups of mobile autonomous agents using nearest neighbor rules," IEEE Transactions on Automatic Control, vol. 48, no. 6, pp. 988-1001, 2003.

[5] R. Olfati-Saber and R. M. Murray, "Consensus problems in networks of agents with switching topology and time-delays," IEEE Transactions on Automatic Control, vol. 49, no. 9, pp. 15201533, 2004.

[6] J. Cortes and F. Bullo, "Coordination and geometric optimization via distributed dynamical systems," SIAM Journal on Control and Optimization, vol. 44, no. 5, pp. 1543-1574, 2003.
[7] J. R. Lawton and R. W. Beard, "Synchronized multiple spacecraft rotations," Automatica, vol. 38, no. 8, pp. 1359-1364, 2002.

[8] S.-J. Lee, K.-K. Oh, and H.-S. Ahn, "Passivity-based output synchronisation of port-controlled Hamiltonian and general linear interconnected systems," IET Control Theory \& Applications, vol. 7, no. 2, pp. 234-245, 2013.

[9] X.-G. Yan, S. K. Spurgeon, and C. Edwards, "Decentralised stabilisation for nonlinear time delay interconnected systems using static output feedback," Automatica, vol. 49, no. 2, pp. 633641, 2013.

[10] X.-G. Yan, S. K. Spurgeon, and C. Edwards, "Global decentralised static output feedback sliding-mode control for interconnected time-delay systems," IET Control Theory and Applications, vol. 6, no. 2, pp. 192-202, 2012.

[11] R. Olfati-Saber, J. A. Fax, and R. M. Murray, "Consensus and cooperation in networked multi-agent systems," Proceedings of the IEEE, vol. 95, no. 1, pp. 215-233, 2007.

[12] Q.-Z. Huang, "Consensus analysis of multi-agent discrete-time systems," Acta Automatica Sinica, vol. 38, no. 7, pp. 1127-1133, 2012.

[13] Y.-Q. Wu, H. Su, and Z.-G. Wu, "Asymptotical synchronization of chaotic Lur'e systems under time-varying sampling," Circuits, Systems, and Signal Processing, vol. 33, no. 3, pp. 699-712, 2014.

[14] Y.-Q. Wu, H. Su, and Z.-G. Wu, "Synchronisation control of dynamical networks subject to variable sampling and actuators saturation," IET Control Theory \& Applications, vol. 9, no. 3, pp. 381-391, 2015.

[15] Y. Hong, G. Chen, and L. Bushnell, "Distributed observers design for leader-following control of multi-agent networks," Automatica, vol. 44, no. 3, pp. 846-850, 2008.

[16] J. Fu, H. Zhang, T. Ma, and Q. Zhang, "On passivity analysis for stochastic neural networks with interval time-varying delay," Neurocomputing, vol. 73, no. 4-6, pp. 795-801, 2010.

[17] J. Hu and Y. Hong, "Leader-following coordination of multiagent systems with coupling time delays," Physica A: Statistical Mechanics and its Applications, vol. 374, no. 2, pp. 853-863, 2007.

[18] Z. Meng, W. Ren, Y. Cao, and Z. You, "Leaderless and leaderfollowing consensus with communication and input delays under a directed network topology," IEEE Transactions on Systems, Man, and Cybernetics Part B: Cybernetics, vol. 41, no. 1, pp. 75-88, 2011.

[19] Y. Hong, J. Hu, and L. Gao, "Tracking control for multiagent consensus with an active leader and variable topology," Automatica, vol. 42, no. 7, pp. 1177-1182, 2006.

[20] X. Wang, W. Ni, and X. Wang, "Leader-following formation of switching multirobot systems via internal model," IEEE Transactions on Systems, Man, and Cybernetics, Part B: Cybernetics, vol. 42, no. 3, pp. 817-826, 2012.

[21] S.-C. Liu, D.-L. Tan, and G.-J. Liu, "Robust leader-follower formation control of mobile robots based on a second order kinematics model," Acta Automatica Sinica, vol. 33, no. 9, pp. 947955, 2007.

[22] C. I. Byrnes, I. G. Lauko, D. S. Gilliam, and V. I. Shubov, "Output regulation for linear distributed parameter systems," IEEE Transactions on Automatic Control, vol. 45, no. 12, pp. 22362252, 2000.

[23] R. Li and H. K. Khalil, "Nonlinear output regulation with adaptive conditional servocompensator," Automatica, vol. 48, no. 10, pp. 2550-2559, 2012.

[24] Y. Su and J. Huang, "Cooperative output regulation of linear multi-agent systems by output feedback," Systems and Control Letters, vol. 61, no. 12, pp. 1248-1253, 2012. 
[25] P. Wieland, R. Sepulchre, and F. Allgwer, "An internal model principle is necessary and sufficient for linear output synchronization," Automatica, vol. 47, no. 5, pp. 1068-1074, 2011.

[26] R. Marino and P. Tomei, "Output regulation for linear systems via adaptive internal model," IEEE Transactions on Automatic Control, vol. 48, no. 12, pp. 2199-2202, 2003.

[27] J. Liu, Z. Liu, and Z. Chen, "Coordinative control of multiagent systems using distributed nonlinear output regulation," Nonlinear Dynamics, vol. 67, no. 3, pp. 1871-1881, 2012.

[28] Y. Su and J. Huang, "Cooperative output regulation with application to multi-agent consensus under switching network," IEEE Transactions on Systems, Man, and Cybernetics, Part B: Cybernetics, vol. 42, no. 3, pp. 864-875, 2012.

[29] X. Wang, Y. Hong, J. Huang, and Z.-P. Jiang, "A distributed control approach to a robust output regulation problem for multi-agent linear systems," IEEE Transactions on Automatic Control, vol. 55, no. 12, pp. 2891-2895, 2010.

[30] H. Su, Y. Wu, and Z. Wu, "Robust output synchronisation of non-identical linear agents via internal model principle," IET Control Theory and Applications, vol. 9, no. 12, pp. 1755-1765, 2015.

[31] Y. Su and J. Huang, "Cooperative output regulation of linear multi-agent systems," IEEE Transactions on Automatic Control, vol. 57, no. 4, pp. 1062-1066, 2012.

[32] H. Liang, H. Zhang, Z. Wang, and J. Wang, "Consensus robust output regulation of discrete-time linear multi-agent systems," Automatica Sinica, vol. 1, no. 2, pp. 204-209, 2014.

[33] H. Liang, H. Zhang, Z. Wang, and J. Wang, "Output regulation of state-coupled linear multi-agent systems with globally reachable topologies," Neurocomputing, vol. 123, pp. 337-343, 2014. 


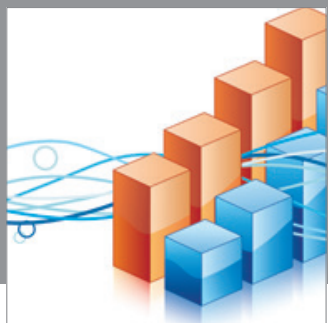

Advances in

Operations Research

mansans

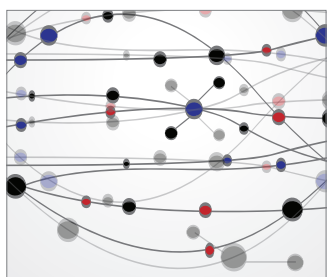

The Scientific World Journal
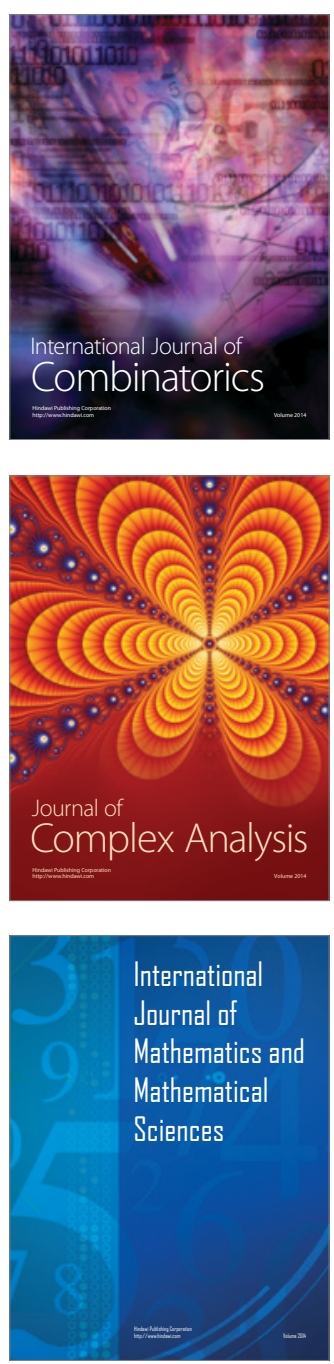
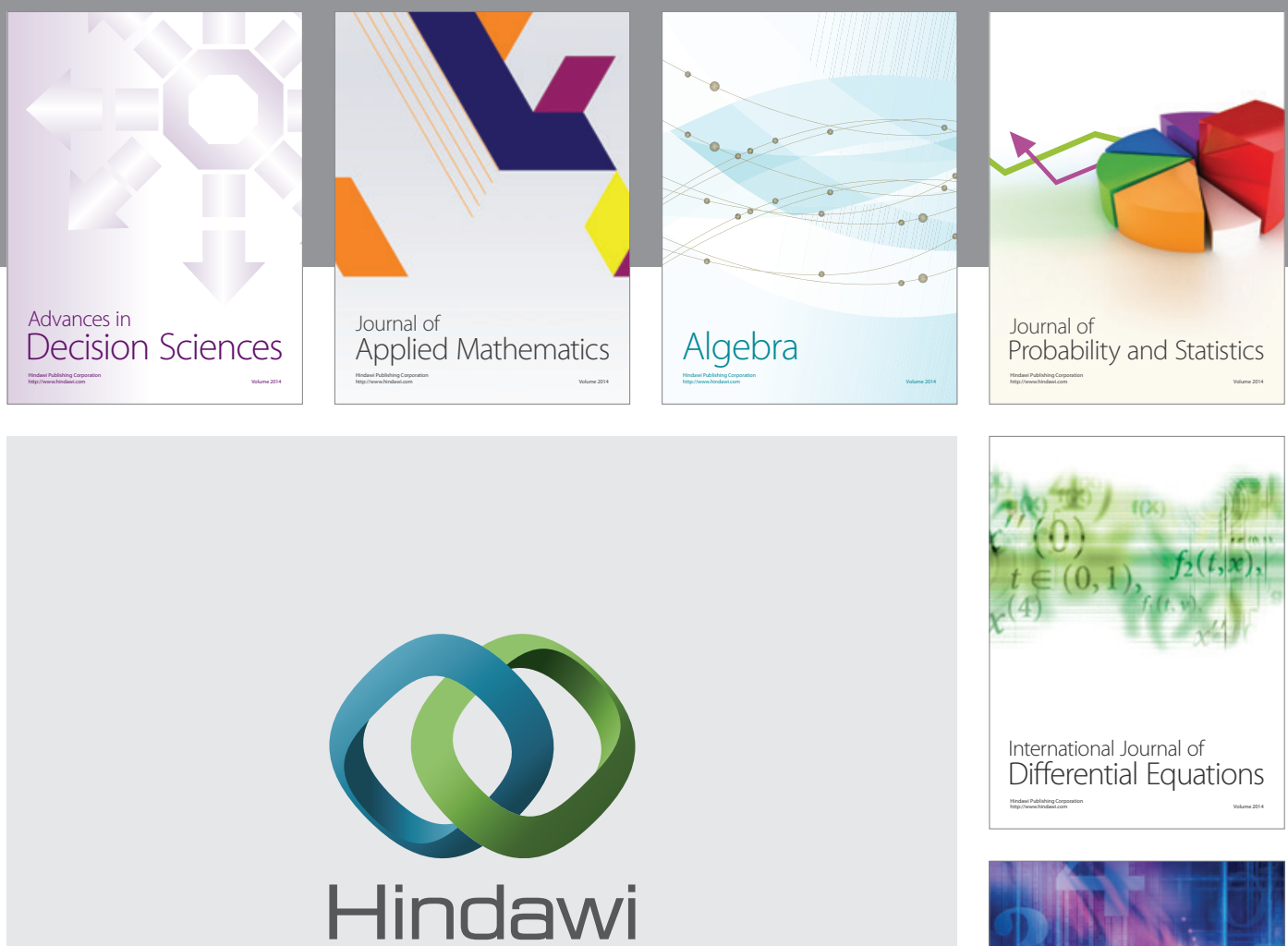

Submit your manuscripts at http://www.hindawi.com
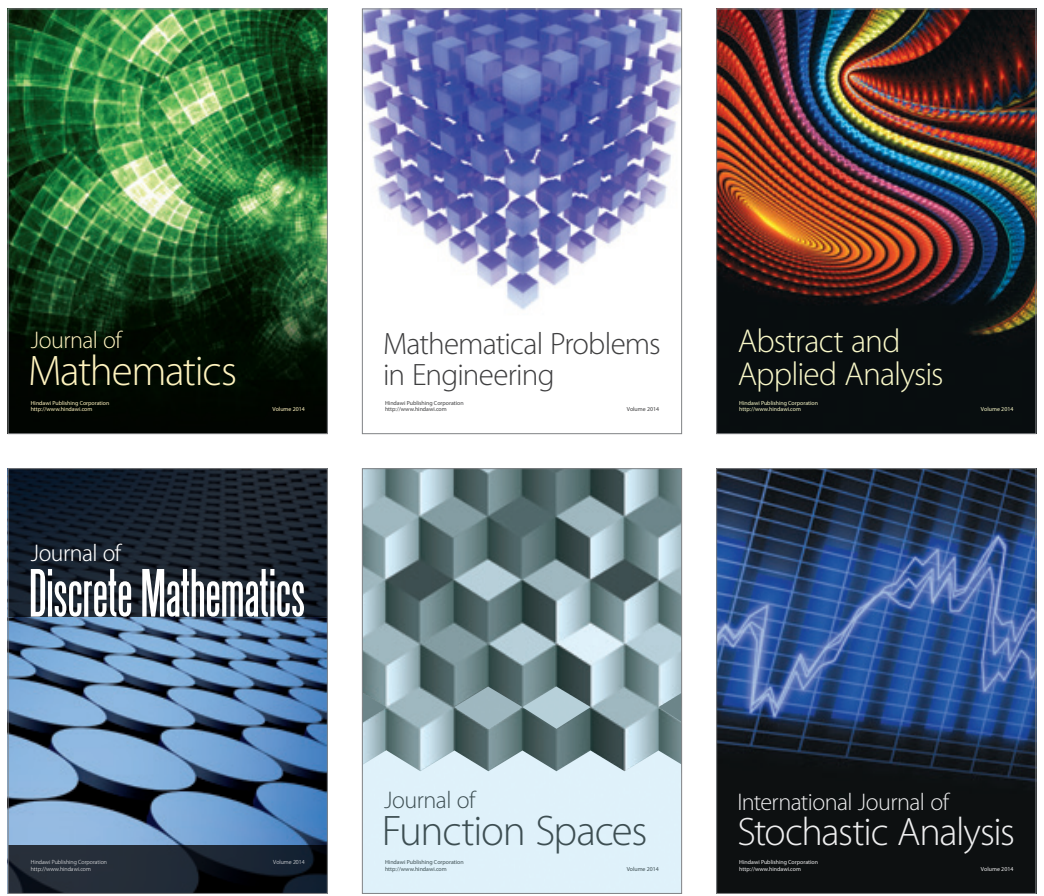

Journal of

Function Spaces

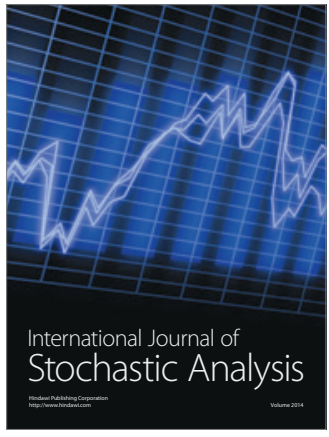

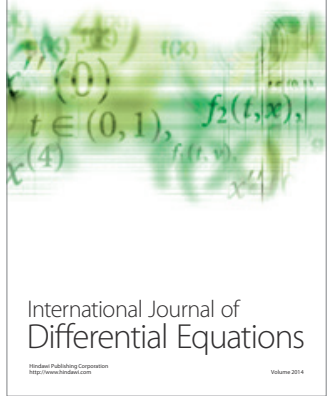
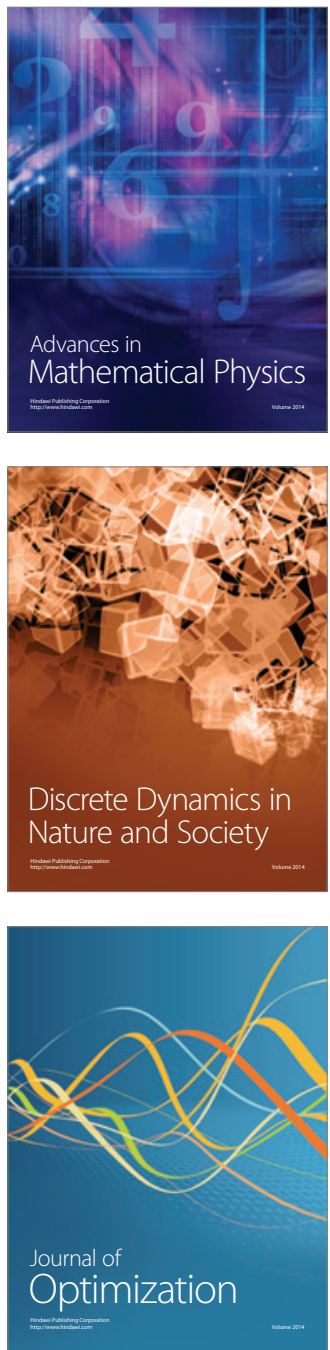